Section Editor Mitchell S.V. Elkind, MD, MS

Camelia Musleh, MD

Lara Marcuse, MD

Madeline Fields, MD

Correspondence to

Dr. Musleh:

camelia.musleh@gmail.com

Mystery Case:

\title{
A young boy with myoclonic jerks
}

\section{SECTION 1}

A 16-year-old right-handed boy presented for consultation for recent development of seizures. He is the product of a normal pregnancy and delivery without a history of developmental delay, head trauma, or family history of epilepsy. A year and a half prior to our consultation, the patient noted that his hand would suddenly jerk across the page while writing in his morning classes. Stress would exacerbate the jerks. Six months later, after staying up late the previous night, the patient awoke early and began to play on the computer when both of his hands suddenly jerked multiple times. He then fell over and began seizing. His mother witnessed tonic contraction of his body followed by clonic activity. He was brought by ambulance to the nearest hospital where a CT head was reported normal.

The patient had a routine EEG at an outside institution and was diagnosed with juvenile myoclonic epilepsy (JME). Valproic acid was started; however, despite increasing dosages, his jerks persisted. The patient then developed episodes of seeing blue, red, and white lights in his right eye followed by right-sided headaches. He underwent video EEG monitoring and was told that these visual symptoms were seizures. His valproic acid was increased and levetiracetam was added. Despite these medications, his jerks and visual seizures continued. Upon consultation with other neurologists, who all believed that the patient had JME, topiramate was added without clinical improvement. Immediately prior to the consultation at this institution, the patient had another generalized tonic-clonic seizure while attending an economics conference. Despite having been a straight-A high school student, he began to have increased difficulty with cognition and his mother pulled him out of school.

Physical examination revealed a well-appearing boy. Mental status was notable for difficulty with serial $7 \mathrm{~s}$ and spelling backwards. Cranial nerve examination was normal. Frequent twitches were noted around his mouth and eyes. Strength was full and sensation was intact to all modalities. Coordination, reflexes, and gait all were normal.

\section{Questions for consideration:}

1. What is the differential diagnosis? Is it possible that the patient has something other than JME?

2. What is the next step in management for this patient? What testing would you order? 


\section{SECTION 2}

Given the patient's age and history of intermittent jerks in the early morning hours, treatmentresistant JME remained high on the differential. However, the presence of cognitive slowing and unusual visual seizures in combination with myoclonic and generalized seizures suggests possible progressive myoclonic epilepsy (PME). Common PMEs include Lafora body disease, UnverrichtLundborg disease (Baltic myoclonus), myoclonic epilepsy with ragged-red fibers (MERRF), neuronal ceroid lipofuscinosis, sialidoses, and dentatorubral-pallidoluysian atrophy. Therefore, a video EEG was ordered with sleep deprivation and photic stimulation. MRI brain was ordered. Valproic acid levels were checked to ensure a therapeutic level.
Video EEG showed moderate generalized slowing with frequent right-greater-than-left posterior spikes and very frequent bursts of diffuse $5-8 \mathrm{~Hz}$ polyspike and wave activity (figure). The patient displayed brief, nearly imperceptible pauses and difficulty with comprehension during the generalized polyspikes. In addition, he had occasional myoclonic jerks preceded by a polyspike. Complaints of "having a thing in my eye" corresponded to a 20-second right occipital seizure. Valproic acid level was therapeutic at $89 \mu \mathrm{g} / \mathrm{mL}$. MRI brain showed some occipital atrophy.

\section{Questions for consideration:}

1. What are the typical EEG findings of JME? What do the patient's EEG findings suggest?

2. What additional tests would you order based on his clinical story and these EEG findings?

Figure EEG with moderate generalized background slowing 4-6 $\mathrm{Hz}$ (rectangle), right-greater-than-left posterior spikes (triangles), and diffuse $5-8 \mathrm{~Hz}$ polyspike and wave (arrow)

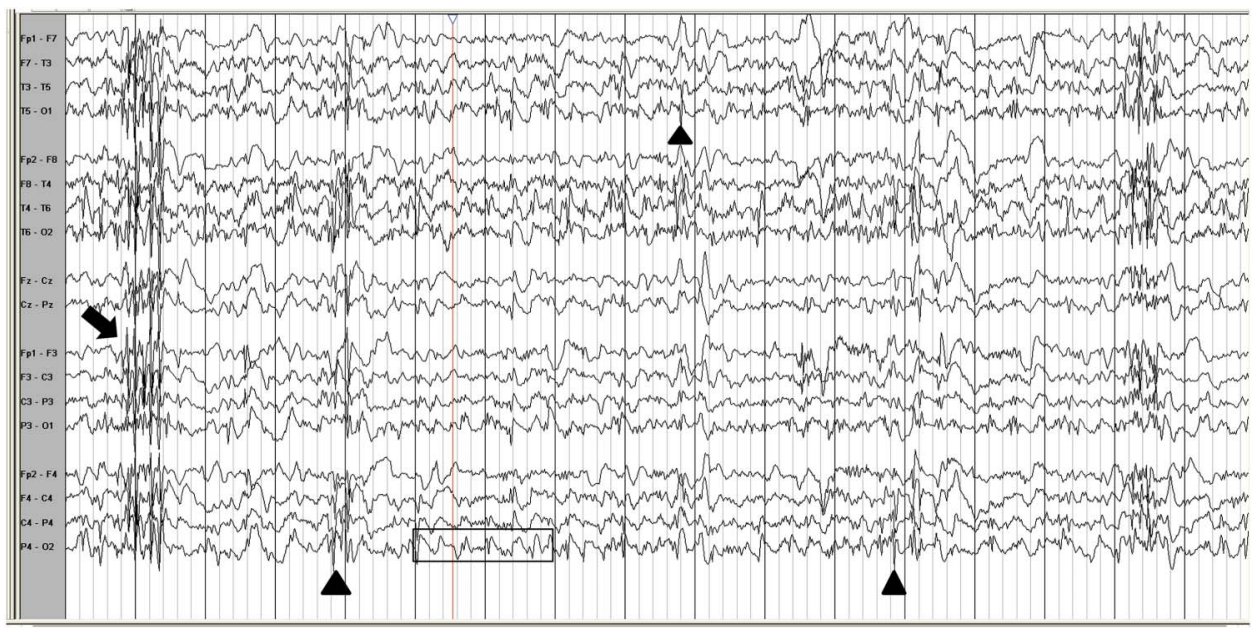




\section{SECTION 3}

Typical interictal EEG findings in JME consist of an irregular mixture of 3- to $6-\mathrm{Hz}$ spikes, polyspikes, or slow waves with fragmentation on top of a normal background with ictal generalized bursts of multiple spikes during myoclonic jerks. ${ }^{1}$ The patient's EEG showed moderate generalized slowing of the background in addition to spikes over the posterior regions (figure). The presence of EEG background slowing can help distinguish between PME and JME since it is present in PME and not in JME. However, certain PMEs such as Unverricht-Lundborg and Lafora body disease can often present with a normal EEG background early in the disease course, thus leading to an initial misdiagnosis of JME. ${ }^{2}$ A way to differentiate Lafora disease from the other PMEs is the presence of occipital seizures with visual phenomena and the presence of spikes over the posterior regions on an EEG. ${ }^{3}$ Thus, genetic testing was sent and skin biopsy was performed to evaluate for Lafora bodies. The skin biopsy was negative for Lafora bodies. However, genetic testing revealed homozygous deletions in the $E P M 2 A$ gene.

\section{Question for consideration:}

1. What treatments are available for Lafora body disease? 


\section{SECTION 4}

Antiepileptic drugs (AEDs) remain the standard therapeutic treatment for Lafora body disease. The first-line agent remains valproic acid given its usual effectiveness against myoclonus and generalized tonic-clonic seizures. AEDs such as levetiracetam, zonisamide, lamotrigine, topiramate, and benzodiazepines may also be helpful. One should avoid phenytoin, carbamazepine, and gabapentin given their ability to potentially worsen myoclonus. ${ }^{4}$ The ketogenic diet has been tested in a small pilot study of Lafora body patients but has not been shown to slow progression. ${ }^{5}$ Since Lafora body disease is known to be resistant and progressive despite aggressive AED management, treatment remains largely palliative.

Since the initial consultation, the patient's seizure frequency worsened and his cognition declined. $\mathrm{He}$ was unable to graduate from high school despite home tutoring. High-dose lorazepam monotherapy has proven to be the most effective with the fewest side effects. He is currently wheelchair-bound and has frequent myoclonic and generalized seizures.

Interestingly, the patient's myoclonus was observed to be more symptomatic during the day than at nighttime. His mother notes that when she covers his eyes she can often lessen the myoclonic jerks and abort a generalized convulsion. She has taken to patching his left eye in order to help stop the jerking and seizures. He was evaluated at our institution by neuro-ophthalmology and given 3 pairs of FL-41 glasses to try at home: dark pink, light yellow, and regular dark. His mother reported that his myoclonus was somewhat better with the regular dark glasses but remarkably improved with the dark pink glasses.

DISCUSSION Lafora body disease is one form of PME. PMEs are a group of disorders characterized by focal and generalized seizures, myoclonus, and progressive neurologic dysfunction, usually with cognitive deterioration and ataxia. ${ }^{6}$ The other common PMEs include Unverricht-Lundborg disease (Baltic myoclonus), MERRF, neuronal ceroid lipofuscinosis, sialidoses, and dentatorubral-pallidoluysian atrophy.

Lafora disease has a typical onset between 12 and 17 years of age. Before onset, children are typically of normal intelligence and physical development. ${ }^{7}$ This can make initial diagnosis difficult to distinguish from typical idiopathic generalized epilepsy. Seizure types can include myoclonus, occipital seizures with transient blindness or visual hallucinations, atypical absences, and atonic and complex partial seizures. Dysarthria and ataxia can also present early on in the disease. Cognitive decline accelerates and dementia occurs later in the disease. As the frequency and severity of seizures worsen, status epilepticus can occur. Many individuals die within a decade from diagnosis, typically from status epilepticus or aspiration pneumonia. ${ }^{4}$

Early on, EEGs show a well-organized background with multiple spike and wave activity and photosensitivity. However, as the disease progresses, the EEG will show an abnormally slow background with loss of sleep features. Bursts of multifocal spike and wave discharges are seen with an occipital predominance. The spike and wave pattern changes from a frequency of $3 \mathrm{~Hz}$ to $6-12 \mathrm{~Hz} .^{4}$

Lafora body disease is an autosomal recessive disorder characterized by mutations in the EPM $2 A$ gene encoding laforin phosphatase or the $E P M 2 B$ gene encoding malin ubiquitin E3 ligase. ${ }^{8}$ The malfunction of these genes results in the formation of abnormal glycogen that accumulates in the brain called Lafora bodies. Thus, Lafora bodies are polyglucan inclusion bodies within neurons that stain positive with periodic acid-Schiff. Lafora bodies can also be found in liver, muscle, sweat glands, and apocrine myoepithelial cells, though it is asymptomatic in these organs. A skin biopsy of the axilla can aid in diagnosis; however, it does not have perfect sensitivity. ${ }^{7}$ Genetic testing shows mutations in the EPM $2 A$ or EPM2B genes.

Treatment includes AEDs such as valproic acid, benzodiazepines, zonisamide, and levetiracetam. Again, one should avoid myoclonus-aggravating drugs such as phenytoin, carbamazepine, and gabapentin. In this patient, it was interesting to note the improvement in myoclonus with patching of one eye and the use of pink lenses. The use of colored glasses may provide some relief for those with photosensitive epilepsy, although this is controversial. ${ }^{9}$ Given the natural history of the disease, it is expected that the patient's seizure frequency and severity will continue to worsen despite treatment. Lafora disease is progressive and fatal usually within 10 years after clinical onset. Genetic counseling as well as social and psychological support should be offered to families.

\section{AUTHOR CONTRIBUTIONS}

Camelia Musleh: drafting/revising the manuscript, analysis or interpretation of data, accepts responsibility for conduct of research and final approval, acquisition of data. Lara Marcuse: drafting/revising the manuscript, analysis or interpretation of data, accepts responsibility for conduct of research and final approval, study supervision. Madeline Fields: drafting/revising the manuscript, study concept or design, analysis or interpretation of data, accepts responsibility for conduct of research and final approval, acquisition of data, study supervision.

\section{ACKNOWLEDGMENT}

The authors thank neuro-ophthalmologist Dr. Janet Rucker for her participation in the care of this patient.

\section{STUDY FUNDING}

No targeted funding reported.

\section{DISCLOSURE}

The authors report no disclosures relevant to the manuscript. Go to Neurology.org for full disclosures. 


\section{REFERENCES}

1. Guerrini R, Bonanni P, Marini C, et al. The myoclonic epilepsies. In: Wyllie E, Gupta A, Lachhwani DK, eds. The Treatment of Epilepsy. Philadelphia: Lippincott Williams \& Wilkins; 2006:407-429.

2. Chan S, Lee W. Benign epilepsy in children. J Formos Med Assoc 2011;110:134-144.

3. Ponsford S, Pye IF, Elliot EJ. Posterior paroxysmal discharge: an aid to early diagnosis in Lafora disease. J R Soc Med 1993;86:597-599.

4. Shahwan A, Farrell M, Delanty N. Progressive myoclonic epilepsies: a review of genetic and therapeutic aspects. Lancet Neurol 2005;4:239-248.

5. Cardinali S, Canafoglia L, Bertoli S, et al. A pilot study of a ketogenic diet in patients with Lafora body disease. Epilepsy Res 2006;69:129-134.

6. Zupanc ML, Legros B. Progressive myoclonic epilepsy. The Cerebellum 2004;3:156-171.

7. Minassian B. Lafora's disease: towards a clinical, pathologic, and molecular synthesis. Pediatr Neurol 2001;25:21-29.

8. Turnbull J, Girard J, Lohi H, et al. Early-onset Lafora body disease. Brain 2012;135:2684-2698.

9. Wilkins A, Baker A, Amin D, et al. Treatment of photosensitive epilepsy using coloured glasses. Seizure 1999;8:444-449.

MYSTERY CASE RESPONSES The Mystery Case series was initiated by the Neurology ${ }^{\circledR}$ Resident \& Fellow Section to develop the clinical reasoning skills of trainees. Residency programs, medical student preceptors, and individuals were invited to use this Mystery Case as an educational tool. Responses were solicited through a group e-mail sent to the American Academy of Neurology Consortium of Neurology Residents and Fellows and through social media. All the answers that we received came through social media, from individ- uals rather than groups. Most of the respondents (42\%) correctly indicated Lafora disease as the most likely diagnosis. Other considerations included JME (33\%) and the idiopathic occipital epilepsy of Gastaut (10\%). The key element was the identification of a slow posterior dominant rhythm in the EEG recording. While this can occur in any chronic epileptic encephalopathy, it is commonly seen in the progressive disorders, like the PMEs.

The most complete answer came from Dr. Alexis Dallara (Child Neurology Fellow, New YorkPresbyterian University Hospital of Columbia, New York). In her response, she pointed out that Lafora disease "presents with seizures, usually myoclonic, clonic or focal, and often has predominant occipital paroxysms on the EEG." In her differential diagnosis, Dr. Dallara considered the idiopathic occipital epilepsy of Gastaut and the idiopathic photosensitive occipital epilepsy. She correctly indicated that epilepsy with grand mal seizures on awakening, as well as JME, are less likely, due to the presence of occipital paroxysms. This Mystery Case illustrates a rare etiology for epilepsy, although one of the most common PMEs. Lafora disease is a fatal autosomal recessive genetic disorder characterized by the presence of cytoplasmic inclusion bodies, known as Lafora bodies, within neurons, and was named after Gonzalo Rodríguez Lafora (1886-1971), a Spanish neuropathologist.

Dragos A. Nita, MD, PhD, FRCPC

Division of Neurology, The Hospital for Sick Children, University of Toronto, Canada. 


\section{Neurology}

Mystery Case: A young boy with myoclonic jerks

Camelia Musleh, Lara Marcuse and Madeline Fields

Neurology 2013;81;e130-e134

DOI 10.1212/WNL.0b013e3182a9f3fe

This information is current as of October 28, 2013

\section{Updated Information \&} Services

\section{References}

Subspecialty Collections

Permissions \& Licensing

\section{Reprints}

including high resolution figures, can be found at: http://n.neurology.org/content/81/18/e130.full

This article cites 8 articles, 0 of which you can access for free at: http://n.neurology.org/content/81/18/e130.full\#ref-list-1

This article, along with others on similar topics, appears in the following collection(s):

All Epilepsy/Seizures

http://n.neurology.org/cgi/collection/all_epilepsy_seizures All Pediatric

http://n.neurology.org/cgi/collection/all_pediatric

Generalized seizures

http://n.neurology.org/cgi/collection/generalized_seizures

Myoclonus; see Movement Disorders/myoclonus

http://n.neurology.org/cgi/collection/myoclonus_see_movement_disord ers-myoclonus

Information about reproducing this article in parts (figures,tables) or in its entirety can be found online at:

http://www.neurology.org/about/about_the_journal\#permissions

Information about ordering reprints can be found online:

http://n.neurology.org/subscribers/advertise

Neurology ${ }^{\circledR}$ is the official journal of the American Academy of Neurology. Published continuously since 1951, it is now a weekly with 48 issues per year. Copyright () 2013 American Academy of Neurology. All rights reserved. Print ISSN: 0028-3878. Online ISSN: 1526-632X.

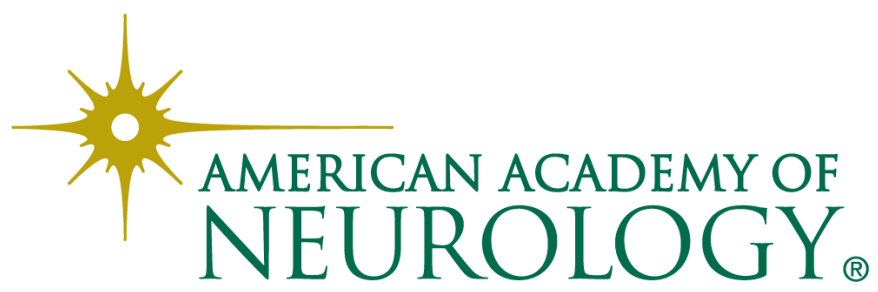

